
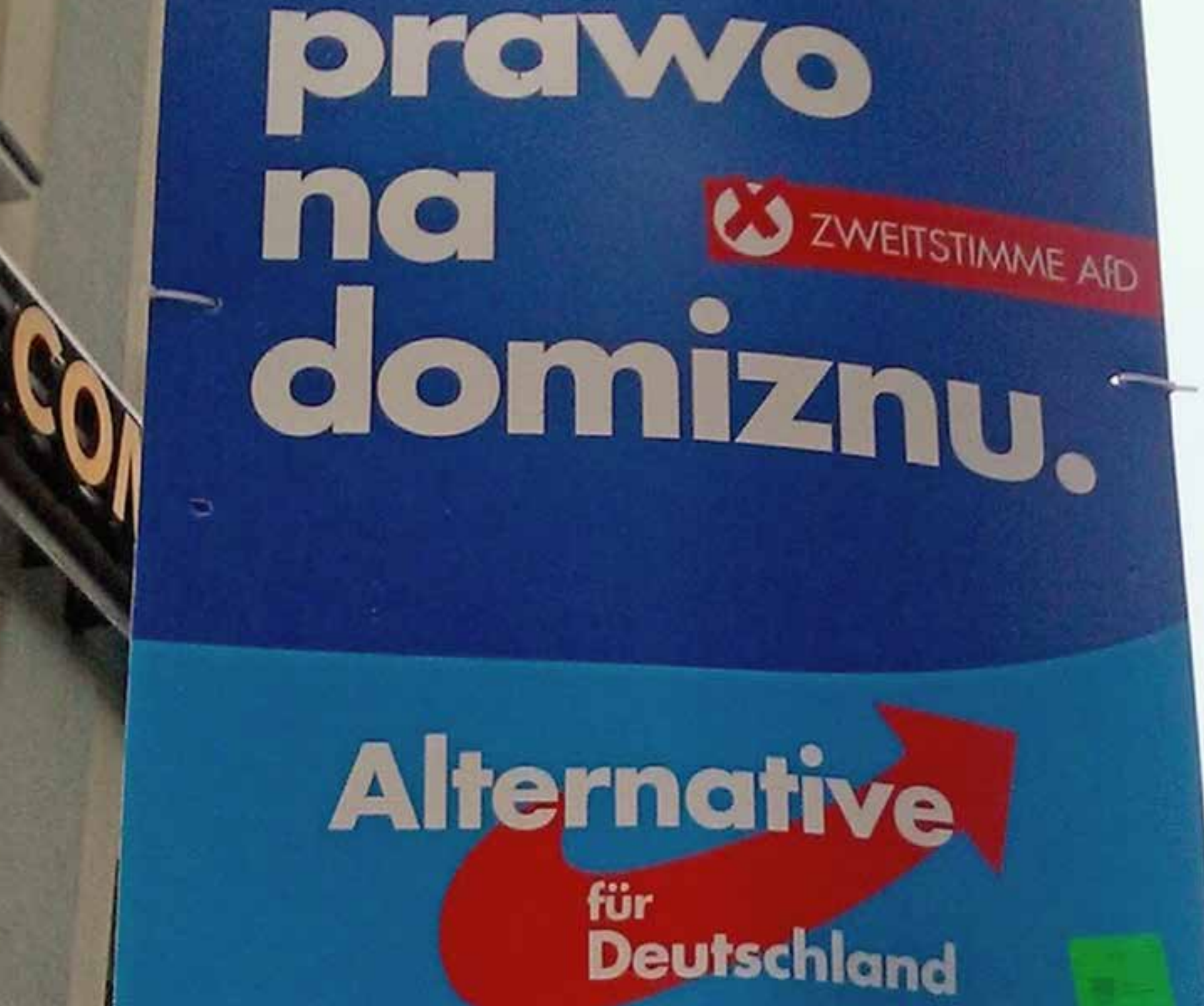

\title{
Ein gescheiterter Staat?
}

\section{Sächsische Besonderheiten}

\author{
Werner J. Patzelt
}

Andersartige Bundesländer oder andersartige Reaktionen?

Lange gab es in Deutschland Diskussionen darüber, ob wohl in Bayern „die Uhren anders gingen“, ja ob ein Bayer deutscher Bundeskanzler werden könne. Auch war, wann immer es in Fernsehen oder Film einen „Deppen vom Dienst“ brauchte, stets ein Bayer gefragt. Das hat sich in der Weise geändert, dass statt Bayern nun Sachsen zum bevorzugten innerdeutschen Verachtungsland geworden ist.
Heute nämlich weiß jeder: Sachsen ist PEGIDA-Gau, Hochburg von Rechtsradikalismus und Rassismus, Inbegriff von Dunkeldeutschland, letztlich ein gescheiterter Staat. Deshalb machen Touristen und Investoren einen weiten Bogen um dieses Land und geben manche Staaten sogar Reisewarnungen heraus, die vom Besuch Dresdens abraten. Auch erkennt man schon an ihrer hässlichen Sprache eine gewisse Beschränktheit der Sachsen - und außerdem daran, dass dieses Völkchen trotz der Befreiung von ostdeut-
Recht auf Heimat: Sorbische Wahlwerbung für die AfD in Hoyerswerda, 2014

(c) Wikimedia (svolks) 
scher Parteidiktatur weiterhin mit großer Mehrheit die CDU wählt.

Oder sind weniger die Sachsen und die Bayern anders, als dass man einfach anders über diese Länder redet als über Brandenburg und NRW? Darauf deutet mancherlei hin. Zudem fragt man sich, ob da nicht oft Neid die Feder führt oder die Sprache prägt. Bayern wie Sachsen schafften es nämlich in Deutschland an die Spitze, wenn auch zu verschiedenen Zeiten und in unterschiedlichen Zusammenhängen. Man nimmt es aber einem Aufsteiger übel, wenn er an seinen Konkurrenten vorbeizieht. Über die rückständigen Bayern machte man sich schon im Bismarckreich lustig - und dann zu bundesdeutschen Zeiten immer noch, als sich der einstige Agrarstaat längst modernisiert hatte, zum High-TechStandort mit führendem Bildungs- und Wissenschaftssystem geworden war, zum Geberstaat unter den Bundesländern, zum innerdeutschen Traumland der Schönen und Reichen sowie zu einer einzigartigen Mischung aus kulturellem Traditionsstolz und technischer Fortschrittslust. So einen Klassenprimus mag man nicht - und erst recht nicht, wenn er andere die eigene Überlegenheit auch fühlen lässt. Spott und üble Nachrede werden dann zur Waffe der weniger Erfolgreichen.

Es mag sein, dass es Sachsen jetzt ähnlich geht. Seit der Wiedervereinigung ist das Land auf stabilem Erfolgskurs. Der hat nicht nur zu schmucken Städten und guten Straßen, sondern auch zu einem ausgeglichenen Staatshaushalt und zu einem Spitzenplatz bei innerdeutschen Bildungsvergleichen geführt. In Sachsen gibt es außerdem unbändigen Stolz auf das eigene Land und auf dessen unübersehbar reiches Kulturerbe. Außerdem fehlt es im weiß-grünen Freistaat nicht am festen Willen, auch aus weiterbestehenden oder neuen Problemen am Ende doch besser herauszukommen als so mancher, der Sachsen jetzt von oben herab kritisiert. Also lässt man Vorwürfe abperlen, die man als ungerechtfertigt empfindet, und geht - ohne das an die große Glocke zu hängen - der Reihe nach jene Fehler an, die zu Recht kritisiert werden.

\section{Einige sächsische Eigentümlichkeiten}

Doch die Ähnlichkeiten zwischen Sachsen und Bayern als den beiden „anderen“ oder „,anders wahrgenommenen“ Ländern Deutschlands enden irgendwo. Es gibt schon das „wirklich Sachsentypische“. Dieses aber lässt sich nicht verstehen ohne den Blick in die Geschichte dieses Landes sowie auf die aus dieser Geschichte empfangenen Prägungen. Sachsen kommt nämlich „von woanders her" und hatte dann auch einen sehr anderen Weg als Bayern. Dieses war seit dem 6. Jahrhundert ein eigenständiges Stammesherzogtum, bestand also längst, bevor von Deutschland in der Geschichte die Rede war. Zwar gab es auch das Stammesherzogtum Sachsen, bevor das Ostfrankenreich zu Deutschland wurde. Doch das erstreckte sich im heutigen Niedersachsen zur Nordsee hin und ist durchaus nicht Vorfahr des heutigen Sachsen. Es wanderte nur dessen Name elbaufwärts und nach Osten.

Das heutige Bundesland Sachsen entstand als Markgrafschaft Meißen in der Grenzregion zum damals noch slawischen Siedlungsgebiet. Im Ergebnis der deutschen Ostsiedlung seit dem 12. Jahrhundert wurde Sachsen dank Landesausbau durch eingewanderte Thüringer und Franken, vor allem aber wegen der Entdeckung von Silber, zu einem der reichsten und mächtigsten deutschen Länder. Die Montanwirtschaft als Macht- und Reichtumsquelle entwickelte sich auch nach dem zweiten „Großen Berggeschrey“ des späten 15. Jahrhunderts weiter. Die Erringung der Kurwürde im späten 14. Jahrhundert, zunächst für das Herzogtum SachsenWittenberg, tat diesen Aufstieg auch noch symbolisch kund. Sich dann obendrein an die Spitze der Reformation stellend und seine Verwaltungssprache über die Luther-Bibel im ganzen deutschen Sprachraum verbreitend, war Sachsen auf einem Gipfel seines Einflusses angelangt.

Doch schon am Ende des 15. Jahrhunderts unterminierte die Leipziger Landesteilung eine dauerhafte Großmachtstellung Sachsens. Stattdessen schuf die mit der Landesteilung einhergehende Machtzersplitterung politischen Raum für den Aufstieg Brandenburg-Preußens. Zwischen diesem werdenden Machtstaat im Norden und dem Habsburgischen Machtblock, der im Süden die böhmischen Länder umfasste, fand sich Sachsen zwei Jahrhunderte später wie ein Pufferstaat eingezwängt. Dafür bezahlte es bis zum Ende der Napoleonischen Zeit als auf Niederlagen abonniertes militärisches Durchzugsland. Zuvor hatten die sächsischen Kurfürsten mit der Erringung der polnischen Königskrone zwischen 1697 und 1763 zwar den Höhepunkt ihres Ansehens erreicht, doch die Möglichkeiten ihres Landes auch überreizt und übernutzt. Die Erschöpfung der Silber- 
vorkommen setzte den politisch begonnenen Abwärtsprozess fort. Das Übrige tat eine oft unglückliche Bündnispolitik.

Neues Selbstbewusstsein schuf freilich die Erhöhung zum eigenen, wenn auch kleinen Königreich nach dem Ende des Alten Reiches. Und neue wirtschaftliche Möglichkeiten eröffnete vor allem die industrielle Revolution. Deren Chancen wusste Sachsen sogar besonders gut zu nutzen. Es baute ein modernes Bildungssystem auf, dessen Schwerpunkt auf moderner Technik durch die Rolle von Dresden und Leipzig als anziehungsmächtigen Kunststädten bestens ausbalanciert wurde. Obendrein schufen Arbeitsamkeit und Fleiß der Bevölkerung, von protestantischem Ethos und praktischer Findigkeit getragen, die Voraussetzungen für große wirtschaftliche Erfolge nicht nur in prosperierenden Städten, sondern zudem in vielen „Industriedörfern“. Ebenfalls dort - und nicht allein in den Ballungsgebieten - entstand die neue, stolze, patriotische und alsbald auch eine politische Modernisierung einfordernde Arbeiterklasse. Am Ende des 19. Jahrhundert war Sachsen dann zum „roten Königreich“ Deutschlands geworden, zum Herzland der SPD, und dabei obendrein - wie schon in der frühen Neuzeit - zur wohlhabendsten Region des Reichs sowie zu einer Heimstatt von Wissenschaft und Kunst. Es war wirklich eine „belle époque“, in der Sachsen damals lebte und sich, samt seinen bonfortionösen auf Sächsisch: „bomforzionösen“ - Verhältnissen, aufrichtig mochte.

Doch unter der Oberfläche gärte es, zumal in den großen Städten, und das nicht nur künstlerisch. Schon zu Weimarer Zeiten traten im zwischen geliebter Tradition und modernem Lebensgefühl zerrissenen Sachsen etliche von Deutschlands politischen Pathologien an die Oberfläche. Bereits im März 1933 wurde der große Dirigent Fritz Busch von den Nazis aus Dresden vertrieben, und bezeichnenderweise fand gleich im September desselben Jahres zu Dresden die erste Ausstellung über „Entartete Kunst“ statt. Obendrein agierte einer der hetzerischsten Nazi-Gauleiter in Sachsen. Der Pöbel war an die Macht gelangt - und fand seine Anhänger quer über die Gesellschaft. Die Zerstörung vieler Innenstädte durch alliierte Bomben war auch dafür die Quittung.

Nach 1945 lebte das einst „rote Königreich“ gleichsam ohne Monarchie und Bourgeoisie wieder auf, ja umfasste bald die gesamte sowjetische Besatzungszone und nachmalige DDR. Insofern war es eine besondere Pointe, dass - nachwirkend bis heute - das Sächsische des aus Leipzig stammenden SED-Führers Ulbricht zu einer Art Signaldialekt des kommunistischen Deutschland wurde. Ihn ironisch anzubringen, wirkte nicht nur im Westen selbsterhöhend und war eine - recht billige - Form von Kritik. Im deutschlandweit beliebten Verächtlichmachen des Sächsischen lebt derlei bis heute nach. Allerdings hatte der Ansehensverlust dieses Dialekts und Tonfalls bereits mit dem realen Machtabstieg Sachsens seit dem späten 18. Jahrhundert begonnen. Als Kennzeichen von Verlierern oder politischen Wirrköpfen deutete man seither das Sächsische immer wieder auch seit 2014 bei PEGIDA.

Umgekehrt begann schon im 18. Jahrhundert das sächsische Aufbegehren gegen Sachsens Verächter. Wer sich zumal seiner Sprache wegen als Dummkopf hingestellt erlebt, der pflegt nämlich zu reagieren. Im schlechten Fall tut er das mit bockiger Verschließung, im besten Fall mit unersättlichem Leistungswillen. Eben der lässt dann die anderen hinter sich und nimmt auf diese Weise an ihnen subtil Rache. Preußens Gloria setzte man jedenfalls Sachsens Glanz, politischer Macht jene Mischung aus Findigkeit und Rührigkeit entgegen, die man auf sächsisch „Fischelanz“ nennt, verballhornt vom lateinisch-französischen Fremdwort „Vigilanz“. Solches Verhalten klingt zwar harmlos, wirkt aber subversiv. Preußen ist jedenfalls vergangen, Sachsen hingegen ein stolzes und erfolgreiches Bundesland. Nicht grundlos begann denn auch das Ende der DDR in den sächsischen Städten Plauen, Dresden und Leipzig - und ebenso das Ende manchen bundesdeutschen Konsenses mit dem Aufkommen von PEGIDA, dem rasch der Aufstieg der AfD folgte.

Politischen Umschichtungen scheinen die Sachsen jedenfalls nicht abgeneigt zu sein, ja sie sogar mitzubewirken, wenn die Verhältnisse gefühlte Fesseln von Fischelanz werden. Nach dem Ende der DDR waren jedenfalls die früheren, so starken sozialdemokratischen Milieus verschwunden. Dafür wurde der sächsische Landesverband der PDS zur zeitweise reformwilligsten Gliederung der alten DDR-Staatspartei. Vor allem aber wählten die Sachsen als neue Staatspartei die CDU über ein Vierteljahrhundert lang ohne Unterbrechung mit absoluten oder starken relativen Mehrheiten in den Landtag und an die Macht. Des Freistaats neuer „republikanischer Monarch“ und erste Ministerpräsident ging dann so weit, we- 
gen der Attraktivität der CDU „seine Sachsen“ für immun gegen jegliche Form von Rechtsextremismus zu erklären. Da aber war die erfolgsverwöhnte CDU bereits selbstgefällig und bräsig geworden und vermochte es nicht mehr, aufsässige Sachsen ins etablierte System zu integrieren. Schon wenig später zog die NPD in den Landtag ein, getragen von einem breiten Netzwerk rechtsradikaler Privatvereinigungen quer übers Sachsenland, die man mitsamt ihren Motivationslagen so recht nicht ernstgenommen hatte. Zehn Jahre darauf entstand in Sachsens Hauptstadt dann PEGIDA - und wurde der weiß-grüne Freistaat zum Spitzenreiter bei Übergriffen auf Geflüchtete und auf deren Unterkünfte. Alsbald war das ehedem rote Sachsen zu Deutschlands braunem Sumpf geworden - und wenn schon nicht in Wirklichkeit, so doch in den Augen von Westdeutschen und von Linken.

Was ist da los? Offenbar können viele Sachsen "links“ und „rechts“ ähnlich gut. Sie wechseln willig die Seiten, wenn es sein muss, sein soll, ja vielleicht auch bloß sein kann. Dabei lassen sie sich nicht weit ins Innere von dem bewegen, was man äußerlich von ihnen will. Man macht mit, bis der innere Kompass anzeigt: „Jetzt is“ aber gutt!“. Denn so gutmütig Sachsen auch sein können, so wenig ist auf handsames Verhalten dann Verlass, wenn sie sich in ausweglose Lagen gebracht fühlen. Das tut eine nennenswerte Minderheit von ihnen seit einiger Zeit bei der Einwanderungspolitik und beim Auftreten des Islam in Deutschland, wenngleich eher beim Vorausblick in die Zukunft als beim Urteil über die Gegenwart. Doch wer fischelant ist, sinnt eben mehr auf Künftiges denn auf das bereits Geschehene. Und er mag es schon gar nicht, wenn mühsam Erreichtes leichtfertig aufs Spiel gesetzt wird - wie in Sachsens Geschichte nicht gerade selten.

\section{Tiefenschichten heutiger politischer Kultur in Sachsen}

Erklärungen sächsischer Eigentümlichkeiten ohne solche historische Tiefenschicht, auch bar des Gespürs für soziokulturelle Zusammenhänge, sind stets unzureichend. Etwa hat die sächsische CDU zweifellos jene rechtsradikalen Netzwerke unterschätzt, die seit Beginn der 1990er Jahre erkennbar waren; doch warum hat sich rechtspopulistisches Empfinden ausgerechnet in jenem Sachsen so verbreitet, das man 1990 als neues sozialdemo- kratisches Musterland erwartete? Gewiss auch verfestigte sich im seit damals von der CDU regierten Sachsen eine rechte Grundstimmung, die den „Kampf gegen rechts“ nicht zum Herzensanliegen der Regierung werden ließ; doch eine rechte Grundstimmung gibt es ebenfalls im seit 1954 von der CSU regierten Bayern, wo - obwohl 2015 von der Zuwanderung am meisten betroffen sich gerade keine nennenswerte Fremdenfeindlichkeit zeigte, sondern bewundernswerte Willkommenskultur. Und sicher sind die Sachsen besonders stolz auf ihr Land; aber das sind die Badener und Württemberger auch, welche die konservativsten aller Grünen an die Macht gewählt haben und wenig Rechtsradikalismus oder Fremdenfeindlichkeit aufweisen.

Man wird also tiefer graben müssen. Dann findet man, dass gerade in Einwanderungsfragen Sachsen viel mehr mit Polen, Tschechien und Ungarn gemein hat als mit den Hansestädten oder dem Rheinland. Was aus westeuropäischer Warte als fragwürdigrechtspopulistisch anmutet, wirkt in Ostmitteleuropa politisch ganz normal - und in Sachsen eben auch. Dort hat man außerdem nicht die Demütigungen durch jene „fremden Vögte" vergessen, als welche viele der seit 1990 ins Land strömenden West-Eliten wirkten. Und heute stört man sich besonders an jenen Denk-, Sprech- und Verhaltensgeboten, die - auf ihre politische Korrektheit so stolze - Wessis gerade in Einwanderungsfragen über „Dunkeldeutschland“ verhängen.

Gerade weil man sich über das erfolgreiche Ende der schweren Umbruchjahre seit 1990 freute, empfand man es als Zumutung, im ,soeben wieder schön gewordenen Sachsen“ jetzt den Ausländeranteil „auf westdeutsches Niveau" bringen zu sollen, also aufs Maß aller Dinge. Weil viele Sachsen - gut gebildet in einem Hochschulland, auch nicht ohne Erfolg mit ihren Handwerksbetrieben, kleinen Geschäften, ja auch mittelständischen Unternehmen - nun einmal kein gutes Ende der seit 2015 betriebenen Einwanderungspolitik erwarten und außerdem empfanden, ihre Kritik werde ,arrogant abgebügelt“, empörten sie sich erst recht. Sie taten das umso mehr, als sie ja nie gefragt wurden, ob sie eine solche Veränderung ihrer Kultur und Heimat wirklich wollten. Derlei „Politik von oben herab“ fühlt sich dann an „wie zu DDR-Zeiten“.

Obendrein fehlen - von perfekt integrierten Vietnamesen abgesehen - jene positiven Erfahrungen mit einer Einwanderungsgesellschaft, die man in weiten Teilen West- 
deutschlands sehr wohl machen konnte. Also schrecken unschöne Bilder, etwa aus Duisburg-Marxloh. So etwas wäre „nicht mehr ihr Land“, empfinden viele. Einige von ihnen machen sich dann ans Abschreckungswerk durch Unwillkommenskultur und Brandanschläge. Weil außerdem die meisten Sachsen zwar derlei Verbrechen ablehnen, doch die so bekämpfte Flüchtlingspolitik auch selbst für falsch erachten, entsteht vielerorts ein Einvernehmenszusammenhang folgender Art: „Das geht zwar zu weit; doch im Grunde ...“. Und weil völlig klar zu sein scheint, dass nur Rechtsextremisten und Rassisten so denken, wird allzu oft in Mithaftung genommen, wer derlei gar nicht ist. Alsbald führt wachsender Trotz zur stillschweigenden Solidarisierung - und fängt ein undifferenzierter „Kampf gegen rechts“ an, die Rechten nur noch weiter zu stärken. Deutschland wiederum findet dann in Sachsen seinen Sündenbock und projiziert auf ihn alle Pathologien unserer Nation.

Tatsächlich werden viele Veränderungen, die auf Deutschlands Einwanderungsgesellschaft zukommen, gerade im landesstolzen Sachsen als drohender Verlust von Beheimatung empfunden. Noch gar nicht lange liegt ja die vom Ende der DDR-Gesellschaft bewirkte Erfahrung zurück, dass man sehr wohl seine Heimat verlieren kann, ohne sie je zu verlassen. Es reicht nämlich, wenn sich die Umwelt über eigene Anpassungswünsche hinaus verändert und man angesichts dessen objektiv machtlos ist. Eben das befürchten jetzt nicht wenige in Sachsen als Folge der von Deutschland passiv hingenommenen Einwanderung, zumal jener aus muslimischen Kulturkreisen. Derzeit wird dieses Gefühl meist als „Rassismus“ etikettiert. Solcher spielt zwar mit, doch eher in einer Nebenrolle. Wichtiger ist, dass man nach über zwanzig Jahren der Systemtransformation das Land Sachsen - nach dessen offenkundigem Niedergang zu DDRZeiten - wieder oben angelangt sieht und nun eine Gefährdung des Erreichten durch zahlenstarke Einwanderung von Migranten mit ganz anderem kulturellen Hintergrund befürchtet. Stark emotionalisierend wirkt in diesem Zusammenhang der oft vorgebrachte Hinweis, erst wenn es „ausreichend viele“ Ausländer und Muslime auch in Sachsen gäbe, hätte die dortige Bevölkerung überhaupt einen Grund, sich - und gar mit Sorgen - in Diskussionen um Deutschlands Einwanderungspolitik einzumischen; jetzt aber halte man besser den Mund und akzeptiere die Politik der Regierung.
Ferner gehört zu den Tiefenschichten derzeitiger sächsischer politischer Kultur ein tief empfundener, doch ungern eingestandener Neid. Meist wird er als „Verlangen nach Gerechtigkeit“ rationalisiert. Dieses wuchs überall im Osten parallel zum Wandel der egalitären DDR-Gesellschaft hin zur auf Konkurrenz und habituelle Distinktion gebauten westlichen Gesellschaft. Als Neid treffen solche Empfindungen heute gerade auch Asylbewerber und Bürgerkriegsflüchtlinge. Die sind nämlich eine weitere, womöglich grenzenlos neu hinzukommende und - angesichts hierzulande nachgefragter Berufskompetenzen - ihre Lage womöglich kaum verbessernde Gruppe von sozialstaatlichen Anspruchsträgern. Unterm Strich entstand so neben der ohnehin schon aufgekommenen Spannungslinie zwischen „oben“ und „unten“ eine weitere Spannungslinie zwischen den „kleinen Leuten“ hierzulande sowie den - wie sie auf Hartz IV-Niveau gestellten - Geflüchteten. Auf diese Weise verbindet sich in brisanter Weise ein materieller Verteilungskonflikt mit den üblichen ethnisch-kulturellen Konflikten einer Einwanderungsgesellschaft.

Er verbindet sich überdies mit der nach der Wiedervereinigung wichtig gewordenen Spannungslinie zwischen eingesessenen Sachsen und hinzugekommenen Westdeutschen. Sie ist zugleich eine Spannungslinie zwischen neuer „kultureller Oberschicht“ und „rückständiger Regionalgesellschaft“. Besonders folgenreich ist sie in Sachsen, weil die dortige Umgangssprache der einzige genuin ostdeutsche Dialekt ist. Während sich alle anderen Regionen der neuen Bundesländer sprachlich mit Gebieten der alten Länder überlappen, lässt sich allein in Sachsen die landfremde Herkunft von Funktionseliten schlicht durch Zuhören feststellen. Diese Spannungslinie wird denn auch in Tausenden von Alltagssituationen immer wieder schmerzlich fühlbar.

Gewiss gab es gute Gründe dafür, die kommunistisch geprägten Funktionseliten der DDR durch Personal aus den alten Bundesländern zu ersetzen. Doch auch eine Generation später haben sich die Sozialräume der hinzugekommenen Westeliten sowie der Einheimischen nicht sonderlich durchmischt, sondern im Wesentlichen nur „überschichtet“. Die „da unten“ sichern als steuerzahlendes oder den Staat subsidiär in Anspruch nehmendes Fußvolk denen „da oben“ ihre Aufgaben und materielle Versorgung, während die „da oben“ allein schon durch 
Präsenz, Dominanz und Habitus zeigen, was alles die „da unten“ noch nicht können oder sind. Das legitimiert dann gleichsam, dass die einen oben, die anderen aber unten bleiben. Aufstieg in die neue Herrenschicht und deren Sozialkreise gelingt zwar durch Bildung, Glück und Anpassung. Wer das alles aber nicht schafft oder sich immerhin der WestAssimilation verweigert, der gilt schnell als rückständig oder allzu arm an jetzt wichtigem kulturellem Kapital. Und wer letzteres wirklich ist, gilt dann rasch als ungehobelter sächsischer Pöbel. Tritt der dann auch noch provokant, ja unübersehbar protestierend auf, dann verdient er wohl nicht mehr als Verachtung und Ausgrenzung. Gegen solches Dunkeldeutschland steht dann Dünkeldeutschland. Ganz wesentlich solchen $\mathrm{Zu-}$ sammenhängen verdankt sich die Eskalationsdynamik um PEGIDA sowie das frühe Großwerden von Rechtspopulismus gerade in Sachsen.

Gegen diesen Hintergrund muss nun auch nicht wundern, dass in Sachsen politische Ohnmachtsempfindungen weit verbreitet sind. Dort aber führten sie vielfach nicht zur Untertänigkeit, sondern gerade zur Aufsässigkeit. Man empfindet, zu wenig Einfluss auf politische Entscheidungen $\mathrm{zu}$ haben und Grundlegendes durch Wahlen ohnehin nicht ändern zu können; also wird es populär, Volksabstimmungen nun auch auf Bundesebene zu fordern. Manche empören sich auch soweit, dass sie die heutige Lage mit der zu DDR-Zeiten gleichsetzen: Schon wieder übersähen Politiker reale Missstände, hörten nicht aufs Volk und ließen die Medien gerade das berichten, was sie selbst sehen und lesen wollten. 1989 hatte man einem solchen Regime auf der Straße sein Ende bereitet; warum also nicht auch jetzt wieder? So entstanden ganz illusorische Hoffnungen, mit Demonstrationen allein könne man in einer repräsentativen Demokratie wie der unseren Weitreichendes verändern.

Zu kurz greift es allerdings, die seit 1990 andauernde CDU-Regierung Sachsens als ursächlich für derzeitige Diktaturvorwürfe auszugeben. Wichtiger sind nachwirkende DDR-Prägungen. Gerade diese, und dabei die frühere Rolle der staatsleitenden Partei mit heutigen vulgärdemokratischen Ansichten kurzschließend, führen etwa zur ziemlich schrägen Ansicht, echte Demokratie gäbe es im Wesentlichen dort, wo die Regierenden einfach nachvollzögen, was man selbst und seinesgleichen für richtig hielte. Wenn angesichts solcher Erwartungen die politische
Klasse nicht demonstrativ zuhören will, ja nicht einmal ihre Politik plausibel erklärt, so entsteht rasch eine wechselseitig verursachte Entfremdungs- und Empörungsspirale. Die ist, trotz fortbestehender CDU-Mehrheiten, auch in Sachsen zu erkennen. Doch während früher die systemkritische PDS solche Stimmungen gegen eine „zu rechte“ Politik auffangen konnte, tut dies bei heutiger Systemkritik ob einer „links-grün versifften“ Politik spiegelbildlich die AfD.

Alle diese Faktoren wirken umso wuchtiger, als auch in Sachsen die zivilgesellschaftlichen Voraussetzungen für eine Bewältigung weiterhin herausfordernder Wandlungsprozesse immer noch recht anders sind als im Westen. Den - in der DDR schwerlich so zu bezeichnenden - „vorpolitischen Raum“ hatte auch in den südlichen Landesbezirken die SED mitsamt ihren Vorfeldorganisationen gründlich durchsetzt. Dabei war die Praxis selbstverantwortlicher Eigeninitiative weitgehend planiert worden. Nach dem Zusammenbruch der SED und ihrer Transmissionsinstitutionen fand sich dann dort, wo eine selbstbewusste Zivilgesellschaft wünschenswert gewesen wäre, ein ruinendurchsetztes Brachland mit nur wenigen tauglichen Fundamenten für einen Neubau. Es ist kein Wunder, dass sich dort - nach dem Ende einer linken Diktatur - orientierungsverheißende rechte Strukturen bildeten.

Obendrein war der SED weitgehend die Entbürgerlichung der einst so reichen und kulturstolzen sächsischen Gesellschaft gelungen. Das lief auf eine Beseitigung der Grundlagen einer selbsttragenden freiheitlichen Ordnung hinaus. Ergänzt wurde derlei um die Beseitigung der wirtschaftlichen Grundlagen eines selbstbewussten Bürgertums, nämlich durch Verstaatlichungen und klassenorientierte Personalpolitik. Seinen Höhepunkt fand das alles in der - solchermaßen nahegelegten und bis zum Mauerbau auch möglichen - Flucht bürgerlicher Elitenangehöriger in den Westen. Das dünnte, zumal im klein- und mittelstädtischen Bereich Sachsens, die demographische Basis einer bürgerlichen Gesellschaft sehr stark aus. Umso leichter war es dann, auch die selbstbewussten Sachsen in ihrem dichtbesiedelten Land auf die Verhaltensanforderungen eines autoritären und paternalistischen Systems zu prägen.

Im Zuge all dessen verschwand jene Rolle, auf die allein sich ein freiheitliches Staatsund Gesellschaftswesen gründen kann, nämlich die eines selbstbewussten, sich nicht 
schwach und staatlicher Stützung bedürftig fühlenden Bürgers. Der nämlich versteht politische Teilhabe nicht vor allem als Fordern und Einfordern, sondern in erster Linie dahingehend, dass er eigene Fähigkeiten, Energie und Zeit bereitwillig in den Dienst anderer stellt. Auch die Friedliche Revolution, obwohl von Sachsen ausgehend und in Sachsen siegend, konnte diese Rolle nicht rasch ins Leben zurückrufen. Einesteils war das ohnehin unmöglich, weil die Etablierung politisch-kultureller Rollen nun einmal längere Entwicklungsprozesse braucht. Andernteils misslang das, weil Revolutionszeiten nun einmal Ausnahmezustände darstellen, die nur schlecht auf gewöhnliche Dauerbelastungen vorbereiten.

Doch auch die folgenden Jahre der demokratischen Konsolidierung Sachsens waren wenig geeignet, eine solche Bürgerrolle flächendeckend neu entstehen $\mathrm{zu}$ lassen. Arbeitslosigkeit grenzte nicht wenige von auf zivilgesellschaftliches Engagement hinführenden Entwicklungsprozessen von vornherein aus. Noch mehr Leute gerieten in eine - oft schockartig erfahrende - Phase der Statusunsicherheit und mussten sich überhaupt eine neue Berufs- und Lebensrolle suchen. Also waren sie mehr mit sich selbst als mit dem von ihnen möglicherweise mitzugestaltenden Gemeinwesen beschäftigt. Und das allzu wenig auf politische Bildung ausgerichtete Schulsystem bringt unter Haupt-, Ober- und Berufsschülern inzwischen allzu viele junge Leute hervor, die sich politisch antiliberal, ja auch rechtsradikal positionieren.

Außerdem braucht dauerhaftes, nicht auf materielle Gratifikationen ausgehendes zivilgesellschaftliches Engagement schon auch ein großes Maß an Selbstbewusstsein und an Stolz auf das bereits Erreichte. An Selbstbewusstsein fehlt es gottlob den Sachsen nicht: Gleich nach dem Ende der SEDHerrschaft fand sich allenthalben das sächsische Weiß-Grün neben deutschem Schwarz-Rot-Gold. Und neu entstehender Stolz richtete sich unmittelbar auf die Rolle Sachsens in der Friedlichen Revolution, mittelbar auf die Glanzzeiten sächsischer Geschichte. $\mathrm{Zu}$ denen wollte man wieder aufschließen. Und weil Kurt Biedenkopf als erster Ministerpräsident des neu entstandenen Freistaates genau das als Vision verkündete und tatkräftig in Regierungspolitik umsetzte, genoss er bald nicht nur großes Vertrauen, sondern regelrechte Verehrung - bis hin zur freundlich-ironischen Rede- weise vom „König Kurt“. Seiner Partei, von einer Reformgruppe um Arnold Vaatz tiefergehend erneuert als andere Landesverbände der ehemaligen Block-CDU, wuchs solches Vertrauen ebenfalls $\mathrm{zu}$ - und wird von Biedenkopfs Nachfolgern inzwischen ratenweise aufgezehrt.

\section{Sachsen-Bashing und seine Folgen}

Eben derlei Selbstbewusstsein und solchen Stolz verletzt das seit dem Medienhype um PEGIDA modisch gewordene „SachsenBashing“ zutiefst. Von ihm profitieren allem Anschein nach auch jene nicht, die es zum Zweck der politischen Auseinandersetzung mit ihren rechtspopulistischen Gegnern in Sachsen selbst betreiben. Kontraproduktiv wird es sogar, wenn es sich bei den Aktivisten des Sachsen-Bashing um Angehörige der neuen Oberschicht mit westdeutschem Migrationshintergrund handelt. Vermutlich wird deren undifferenziert angelegter Kampf gegen das „braune Sachsen“ nur mehr und mehr Sachsen den Rechtspopulisten zutreiben wie anfangs bei PEGIDA und inzwischen bei der AfD.

Derweil geht Sachsen auf eine Phase scharfer innenpolitischer Polarisierung zu. In ihr werden bundesweit wichtige Konflikte exemplarisch ausgetragen, zumal solche um Einwanderung und Islam, um Heimat und Nationalstolz, um Eliten und Demokratie. Sollte Sachsens markante Rolle bei solchen Debatten daher rühren, dass jene Themen sich in anderen Bundesländern leichter vom politischen Diskurs fernhalten lassen als im weißgrünen Freistaat, so dürften dafür die erörterten Besonderheiten Sachsens ursächlich sein, darunter vor allem die sprachlich markante Grenze zwischen angestammter Bevölkerung und landfremden Eliten.

Und am Ende mag sich zeigen, dass die geläufige ironische Charakterisierung von Sachsen als „helle, höflich und heimtückisch“ sich bei alledem wie folgt ausgewirkt hat: Höflich hielten sich die allermeisten von PEGIDAs Pöblern fern, waren aber helle genug, reale Probleme rechtzeitig zu erkennen - und agierten darin heimtückisch gegen ihre Kritiker, dass sie durch Erzwingung öffentlicher Debatten und entsprechendes Wahlverhalten auch auf die Lösung jener Probleme hinwirkten. Wenn das aber wirklich so kommen sollte und Sachsen einmal mehr besonders erfolgreich aus einer Krise hervorginge: Wer wollte derlei Andersartigkeit dann wirklich zum Vorwurf machen!

\section{Autor}

Prof. Dr. Werner J. Patzelt TU Dresden Institut für Politikwissenschaft Professur für Politische Systeme und Systemvergleich 01062 Dresden 